\title{
Nonlinear Optical Interactions and Relaxation in 2D Layered Transition Metal Dichalcogenides Probed by Optical and Photoacoustic Z-scan Methods
}

Melissa E. Maldonado, ${ }^{1}$ Avishek Das, ${ }^{1}$ Ali M. Jawaid, ${ }^{2}$ Allyson J. Ritter, ${ }^{2}$ Richard A Vaia, ${ }^{2}$ Danilo A. Nagaoka, ${ }^{3}$ Pilar G. Vianna, ${ }^{3}$ Leandro Seixas, ${ }^{3}$ Christiano J. S. de Matos ${ }^{3}$, Alexander Baev ${ }^{4}$, P. N. Prasad ${ }^{4}$ and Anderson S. L. Gomes ${ }^{1}$

\section{Supporting Information}

\section{Numerical Integration}

The numerical integration was carried using the adaptive Simpson quadrature implemented in Matlab environment. We integrated the paraxial wave equation

$$
\frac{d I\left(z^{\prime}, z, r\right)}{d z^{\prime}}=-\frac{\alpha_{0} I\left(z^{\prime}, z, r\right)}{1+I\left(z^{\prime}, z, r\right) / I_{s a t}}-\beta I^{2}\left(z^{\prime}, z, r\right)
$$

over the sample thickness and the cross sectional area of the laser beam to find the transmitted power:

$$
P_{\text {out }}(z)=P_{\text {in }}(z)-2 \pi \int_{0}^{\infty} \int_{-L / 2}^{L / 2} r\left(\frac{\alpha_{0} I\left(z^{\prime}, z, r\right)}{1+I\left(z^{\prime}, z, r\right) / I_{\text {sat }}}+\beta I^{2}\left(z^{\prime}, z, r\right)\right) d z^{\prime} d r .
$$

The normalized transmission is then defined as:

$$
T(z)=P_{\text {out }}(z) / P_{0}, \quad T_{\text {norm }}(z)=\frac{T(z)}{1-\alpha_{0} L}, \quad P_{0}=\frac{\pi w_{0}^{2}}{2} I_{0},
$$

where $w_{0}$ is the beam waist radius, $L$ is the sample thickness and $z$ ' is the propagation depth inside the sample.

The following equations were used, from ref [1].

$$
\begin{gathered}
\alpha(I)=\alpha_{0}+\beta I \\
T(z)=\frac{1}{\sqrt{\pi} q(z)} \int_{-\infty}^{\infty} \ln \left[1+q(z) \exp \left(-\tau^{2}\right)\right] d \tau
\end{gathered}
$$

Where: $\quad q(z)=\frac{\beta I_{0} L_{e f f}}{1+\left(z / z_{0}\right)^{2}} \quad$ and $\quad$ Leff $=\frac{1-\exp \left(-\alpha_{0} L\right)}{\alpha_{0}}$ 
If, $q(z) \ll 1$, then $\quad T(z)=\sum_{m=0}^{\infty} \frac{[-q(z)]^{m}}{(m+1)^{3 / 2}}$

Considering now the ground state saturation, the following equations are employed, following ref. [2]:

$$
\begin{gathered}
\alpha(I)=\frac{\alpha_{0}}{1+\frac{I}{I_{s}}}+\beta I \\
T(z)=\frac{Q(z)}{\sqrt{\pi} q(z)} \int_{-\infty}^{\infty} \ln \left[1+q(z) \exp \left(-\tau^{2}\right)\right] d \tau
\end{gathered}
$$

Where $\mathrm{Q}(\mathrm{z})$ and $\mathrm{q}(\mathrm{z})$ are, respectively:

$$
Q(z)=\exp \left(\frac{\alpha_{0} L I}{I+I_{S}}\right) \quad q(z)=\frac{\beta I_{0} L_{e f f}}{1+\left(z / z_{0}\right)^{2}}
$$

Figures S1, S2 and S3 show, respectively, the results from the numerical integration and approximate fits for $\mathrm{MoS}_{2}, \mathrm{NbS}_{2}$ and $\mathrm{ZrTe}_{2}$ at different excitation intensities indicated in each figure.
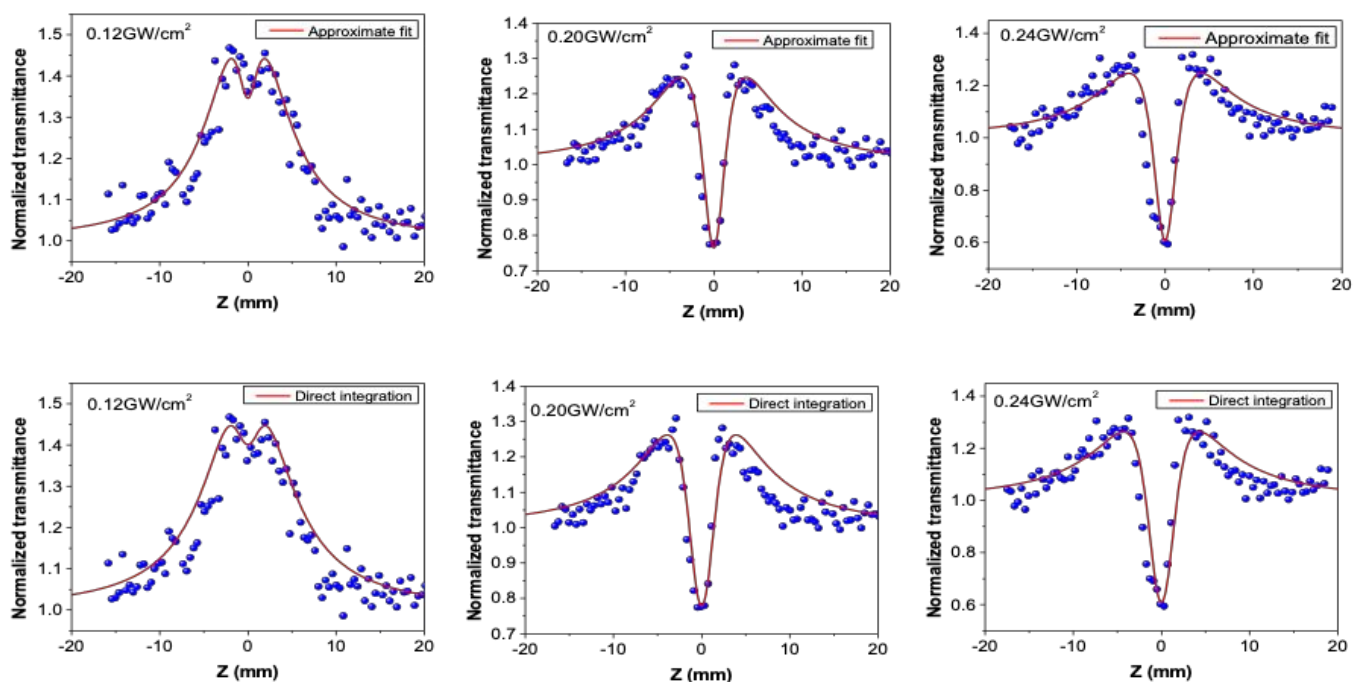

Figure S1 - Approximate fit (upper row, red lines) and direct integration (lower row, red lines) for OZ-Scan in $2 \mathrm{D} \mathrm{MoS}_{2}$ nanoflakes ACN suspension. The blue dots are the experimental results. 

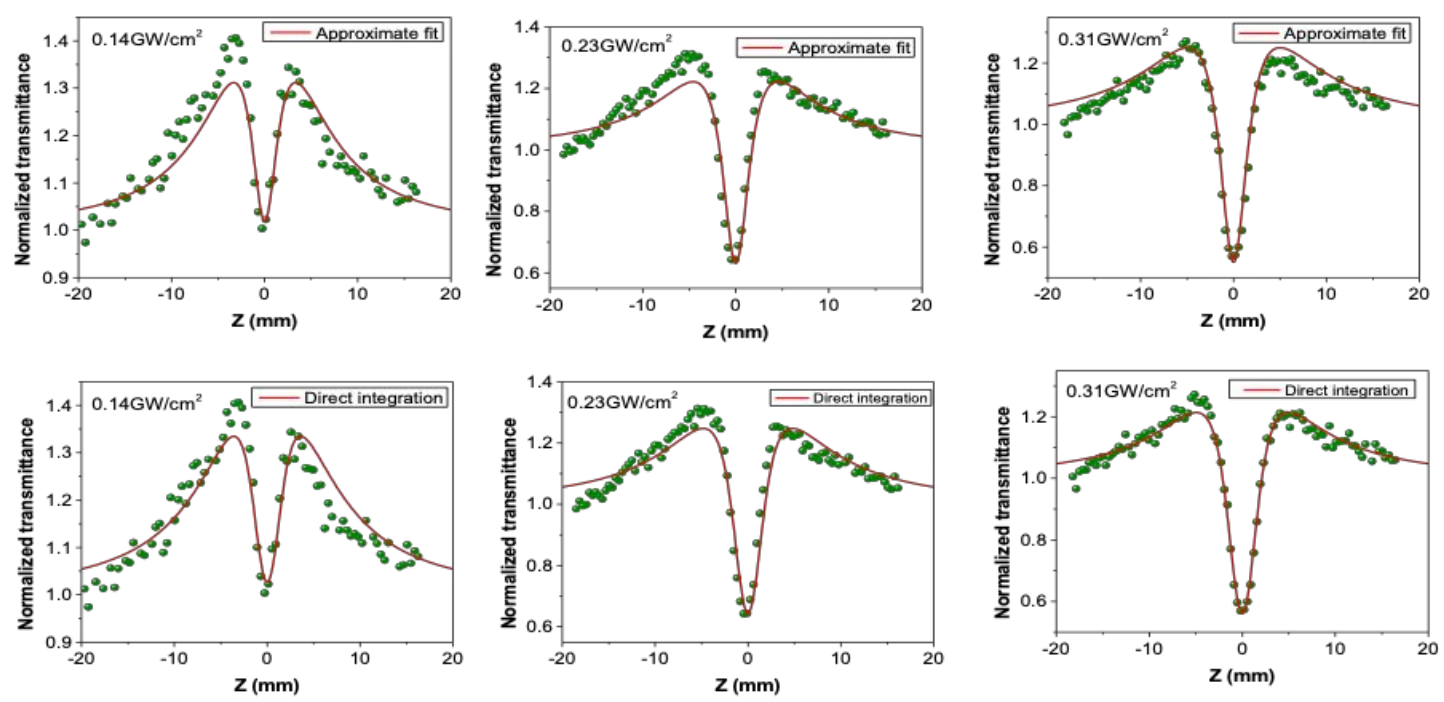

Figure S2 - Approximate fit (upper row, red lines) and direct integration (lower row, red lines) for OZ-Scan in $2 \mathrm{D} \mathrm{NbS}_{2}$ nanoflakes $\mathrm{ACN}$ suspension. The green dots are the experimental results.
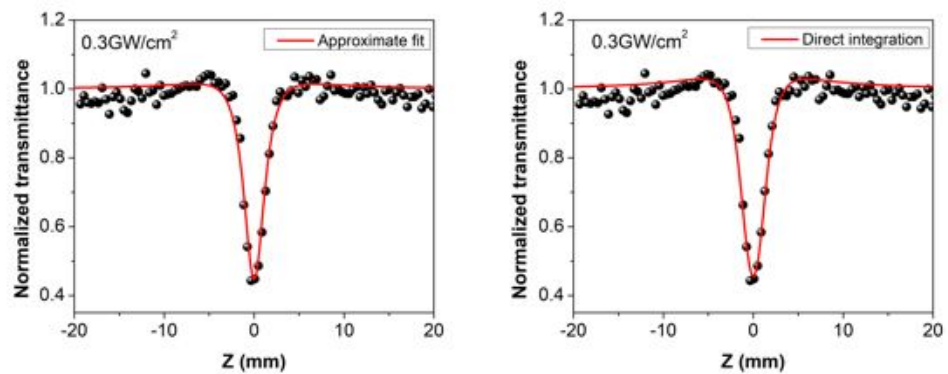

Figure S3 - Approximate fit (left, red lines) and direct integration (right, red lines) for OZ-Scan in 2D $\mathrm{ZrTe}_{2}$ nanoflakes ACN suspension. The green dots are the experimental results. 
The values for the relevant parameters $\mathrm{I}_{\mathrm{SAT}}$ and $\beta$ are given in tables SI-1, SI-2 and SI-3 for $\mathrm{MoS}_{2}, \mathrm{NbS}_{2}$ and $\mathrm{ZrTe}_{2}$, respectively.

Table SI-1 - Calculated $\mathrm{I}_{\mathrm{SAT}}$ and $\beta$ for $\mathrm{MoS}_{2}$

\begin{tabular}{|c|c|c|c|}
\hline \multicolumn{4}{|c|}{$\mathrm{MoS}_{2}$} \\
\hline $\mathrm{I}_{0}\left(\mathrm{GW} / \mathrm{cm}^{2}\right)$ & $\alpha_{0}(\mathrm{~cm}-1)$ & $\beta \times 10^{-7}(\mathrm{~cm} / \mathrm{W})$ & $\mathrm{I}_{\mathrm{SAT}}\left(\mathrm{MW} / \mathrm{cm}^{2}\right)$ \\
\hline \multicolumn{4}{|c|}{ Approximate fit } \\
\hline 0.12 & 2.3 & 0.15 & 12 \\
\hline 0.20 & 2.2 & 0.25 & 17 \\
\hline 0.24 & 2.2 & 0.25 & 17 \\
\hline \multicolumn{4}{|c|}{ Direct integration } \\
\hline 0.12 & 2.6 & 0.33 & 7 \\
\hline 0.20 & 2.6 & 0.53 & 10 \\
\hline 0.24 & 2.6 & 0.53 & 10 \\
\hline
\end{tabular}

Table SI-2 - Calculated $\mathrm{I}_{\mathrm{SAT}}$ and $\beta$ for $\mathrm{NbS}_{2}$

\begin{tabular}{|c|c|c|c|}
\hline \multicolumn{4}{|c|}{$\mathrm{NbS}_{2}$} \\
\hline $\mathrm{I}_{0}\left(\mathrm{GW} / \mathrm{cm}^{2}\right)$ & $\alpha_{0}(\mathrm{~cm}-1)$ & $\beta \times 10^{-7}(\mathrm{~cm} / \mathrm{W})$ & $\mathrm{I}_{\mathrm{SAT}}\left(\mathrm{MW} / \mathrm{cm}^{2}\right)$ \\
\hline \multicolumn{4}{|c|}{ Approximate fit } \\
\hline 0.14 & 2.0 & 0.25 & 8 \\
\hline 0.23 & 1.8 & 0.25 & 10 \\
\hline 0.31 & 1.8 & 0.20 & 10 \\
\hline \multicolumn{4}{|c|}{ Direct integration } \\
\hline 0.14 & 2.3 & 0.57 & 4 \\
\hline 0.23 & 2.1 & 0.54 & 5 \\
\hline 0.31 & 2.1 & 0.42 & 8 \\
\hline
\end{tabular}

Table SI-3 - Calculated $\mathrm{I}_{\mathrm{SAT}}$ and $\beta$ for $\mathrm{ZrTe}_{2}$

\section{$\mathrm{ZrTe}_{2}$}

$I_{0}\left(\mathrm{GW} / \mathrm{cm}^{2}\right) \quad \alpha_{0}(\mathrm{~cm}-1) \quad \beta \times 10^{-7}(\mathrm{~cm} / \mathrm{W}) \quad I_{S A T}\left(\mathrm{MW} / \mathrm{cm}^{2}\right)$

Approximate fit

\begin{tabular}{l|ccc|}
\hline 0.26 & 0.7 & 0.23 & 15 \\
\hline 0.26 & & Direct integration & \\
\hline
\end{tabular}




\section{Density of States}

We calculated the electronic band structure and the projected density of states (PDOS) of $\mathrm{NbS}_{2}$ and $\mathrm{ZrTe}_{2}$ with first-principles methods based on well-developed Density Functional Theory (DFT) [1,2], implemented in SIESTA code [3] to calculate the electronic properties of $\mathrm{NbS}_{2}$ and $\mathrm{ZrTe}_{2}$.

In these PDOS, we note the van Hove singularities (VHS) peaks related to two-photon and one-photon absorption, see fig. S4.
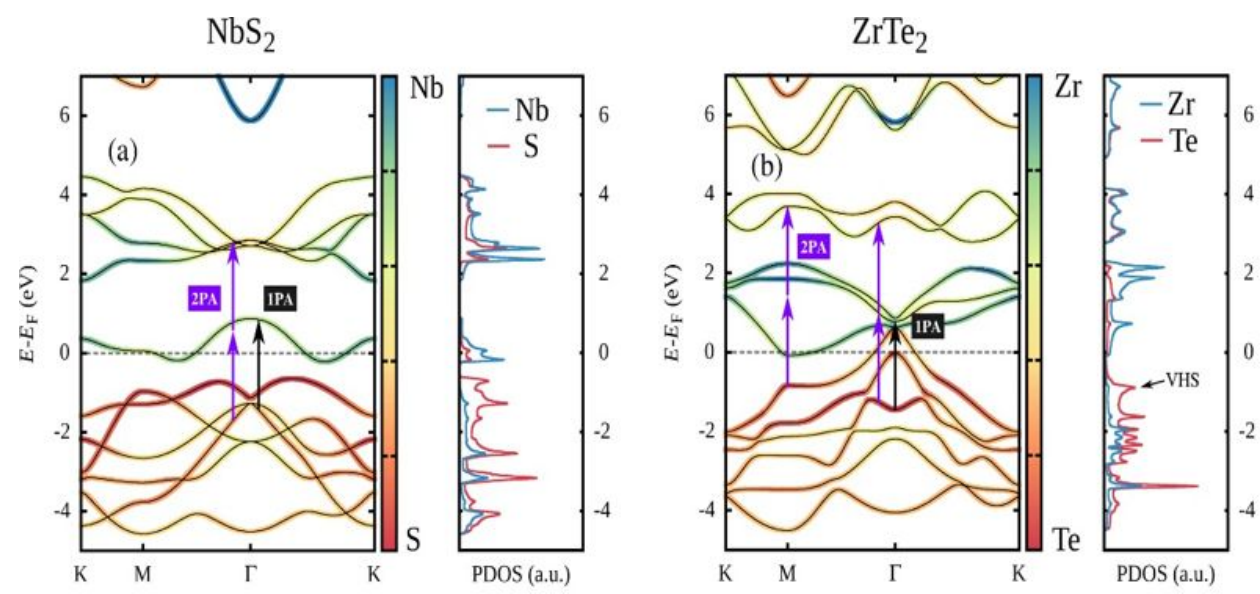

Fig. S4 - Electronic band structure and PDOS of $\mathrm{NbS}_{2}$ and $\mathrm{ZrTe}_{2}$. VHS indicates van Hove singularities.

\section{References}

(1) Hohenberg, P.; Kohn, W. Inhomogeneous Electron Gas. Phys. Rev. 1964, 136 (3B), B864B871.

(2) Kohn, W.; Sham, L. J. Self-Consistent Equations Including Exchange and Correlation Effects. Phys. Rev. 1965, 140 (4A), A1133-A1138.

(3) Soler, J. M.; Artacho, E.; Gale, J. D.; García, A.; Junquera, J.; Ordejón, P.; Sánchez-Portal, D. The SIESTA Method for Ab Initio Order- N Materials Simulation. J. Phys. Condens. Matter 2002, 14 (11), 2745-2779. 